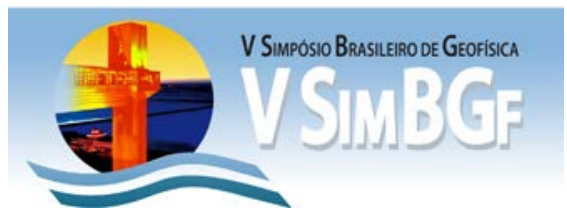

\title{
Topografia do embasamento gravimétrico na região da Bacia de Santos, SP
}

Constantino, R., Molina, E. C., Instituto de Astronomia, Geofísica e Ciências Atmosféricas da Universidade de São Paulo

Copyright 2012, SBGf - Sociedade Brasileira de Geofísica

Este texto foi preparado para a apresentação no V Simpósio Brasileiro de Geofísica, Salvador, 27 a 29 de novembro de 2012. Seu conteúdo foi revisado pelo Comitê Técnico do $V$ SimBGf, mas não necessariamente representa a opinião da SBGf ou de seus associados. É proibida a reprodução total ou parcial deste material para propósitos comerciais sem prévia autorização da SBGf.

\section{Resumo}

O presente trabalho modelou as variações de profundidade da Interface Crosta manto (ICM) e a topografia do embasamento na região da Bacia de Santos a partir de uma análise combinada de dados gravimétricos, batimétricos, modelo de espessura sedimentar e espessura crustal a partir de uma metodologia que envolve inversão gravimétrica e análise flexural. $O$ resultado apresentou a topografia do embasamento com feições de até $700 \mathrm{~m}$, que parecem estar em concordância com feições discutidas em trabalhos pretéritos, como por exemplo, a cadeia vulcânica Avedis. A profundidade do embasamento estimada durante este trabalho mostrou profundidades que vão de 500 à $10500 \mathrm{~m}$. A região mais profunda do embasamento condiz com a falha de Cabo Frio.

\section{Introdução}

O conhecimento do embasamento oceânico é de grande importância em diversas aplicações na área de geociências, pois sua estrutura representa a situação tectônica e a história de uma determinada região.

O fundo oceânico sofre um constante processo de sedimentação. Em algumas áreas, a cobertura de sedimentos pode ocultar feições tectônicas do embasamento como lineamentos, blocos crustais inclinados, cordilheiras e eixos de espalhamento. O conhecimento dessas estruturas pode ser extremamente útil em estudos geofísicos que visam investigar as estruturas da crosta. Por exemplo, em situações onde campanhas de coleta de dados geofísicos são realizadas ao longo de perfis, o ideal é traçá-los ortogonalmente aos lineamentos. Como o embasamento do fundo oceânico ainda não foi globalmente cartografado, grandes erros podem ser gerados durante a locação desses perfis de coleta de dados.

Devido à distribuição global e de boa qualidade, obter dados associados ao campo de gravidade através de altimetria por satélite pode ser um bom método para estudos de larga escala que visam estudar a crosta (Woodward \& Wood , 2000).

Vários trabalhos são encontrados na literatura onde aspectos do fundo oceânico são investigados através de dados de altimetria por satélite. Smith \& Sandwell (1997) integraram dados de navio com dados de altimetria por satélites para construir um mapa da topografia dos oceanos. Ramillien \& Cazanave (1996) usaram dados in situ obtidos do NGDC e dados do ERS1 GEODETIC MISSION para calcular globalmente a batimetria dos oceanos. Calmant \& Baundry (1996) mostraram em seu estudo diferentes técnicas para A obtenção de modelos batimétricos a partir de dados de altimetria por satélite. Durante este trabalho, os dados gravimétricos derivados de altimetria por satélites são utilizados para mapear a topografia do embasamento.

A interpretação do campo gravimétrico referente ao embasamento é importante para auxiliar na interpretação de dados sísmicos ou para fornecer informações em áreas onde tais dados são escassos ou indisponíveis. Isto ocorre na Bacia de Santos, que devido ao seu alto interesse exploratório, não permite a disponibilização de grande parte dos dados coletados e processados por empresas do setor energético.

No presente trabalho foi determinada a configuração do embasamento oceânico na Bacia de Santos através da análise combinada de dados do campo de gravidade, da batimetria, da espessura sedimentar e da espessura da crosta na região. Trabalhos pretéritos como de Braitenberg et al. (2006) utilizaram estes dados e metodologia e determinaram com sucesso a estrutura do embasamento oceânico do Mar da China.

\section{Metodologia}

O ponto inicial para o estudo é a base de dados contendo: observações de anomalia da gravidade, obtidas por Molina (2009) através de altimetria por satélite e geofísica marinha; estimativa da espessura crustal obtidas em Leyden (1971) e Zalán (2011), modelo de espessura sedimentar, obtido do banco de dados da NGDC e compilados pela NOAA, e modelo de batimetria global, disponível publicamente pela GEBCO. Todas as etapas deste trabalho foram desenvolvidas com base na metodologia proposta por Braitenberg et al. (2006) e estão descritas a seguir:

Etapa 1 - O objetivo desta etapa é modelar a profundidade da Interface Crosta Manto (ICM). Para isto, o primeiro passo compreende calcular 0 efeito gravimétrico do pacote sedimentar levando em consideração a compactação sedimentar com a profundidade e subtraí-lo da anomalia Bouguer. O campo resultante é invertido a partir do Modelo de Inversão Gravimétrica Iterativo descrito por Braitenberg e Zadro (1999) obtendo assim as ondulação da ICM;

Etapa 2 - Esta etapa tem como objetivo checar a confiabilidade dos dados obtidos na etapa anterior. Para isto, a ICM é calculada a partir de uma metodologia independente, a qual envolve análise flexural. Aqui é calculada a flexura da crosta pela a carga topográfica e intracrustal (referente à camada sedimentar); 
Topografia do embasamento gravimétrico na região da Bacia de Santos, SP

Etapa 3 - Nesta etapa, o efeito gravimétrico da ICM obtida na etapa 2 é calculado pelo algoritmo de Parker (1972). O efeito gravimétrico da ICM mais o efeito gravimétrico dos sedimentos são eliminados da anomalia Bouguer, resultando no campo gravimétrico residual.

Etapa 4 - Nesta etapa, o campo gravimétrico residual referente à topografia do embasamento é invertido ultizando a mesma metodologia proposta na etapa 1 , resultando nas estruturas do embasamento na região da Bacia de Santos.

Todas as etapas foram desenvolvidas com o auxílio do software Lithoflex.

\section{Resultados}

A topografia do embasamento é calculada seguindo as quatro etapas descritas anteriormente. $\mathrm{Na}$ etapa 1 , o efeito gravimétrico dos sedimentos é calculado utilizando o modelo de Sclater \& Christie (1980) em uma série de camadas finas (10m de espessura) com densidade lateral variável. A camada de sedimentos contribui no sinal gravimétrico na ordem de aproximadamente -10 a -30 mGal.

Para a inversão, o comprimento de onda de corte foi estimado a partir do decaimento do espectro de amplitude do campo gravimétrico (Russo \& Speed, 1994) e o valor encontrado foi de $115 \mathrm{~km}$. O campo gravimétrico é invertido para um contraste de densidade lateralmente variável entre a crosta e o manto. Estes valores foram retirados do modelo CRUST 2.0.

O processo de inversão é realizado para uma série de valores de profundidades de referência variando entre 20 e $30 \mathrm{~km}$. O erro RMS é calculado entre os valores obtidos por inversão e vínculos sísmicos obtidos em Leyden (1971) e Zálan, (2011). Após realizados os testes com valores de profundidade de referência variável, a profundidade de $28.7 \mathrm{~km}$ com um erro RMS de aproximadamente $1.9 \mathrm{~km}$ foi a que forneceu o melhor resultado (Figura 1 ).

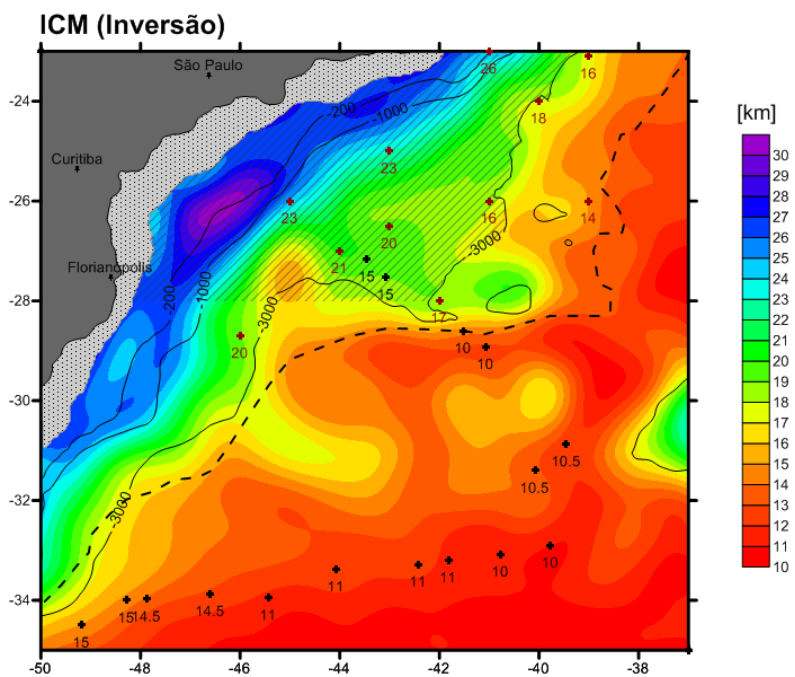

Figura 1: ICM gravimétrica. Em preto, vínculos de Leyden (1971) em preto, vínculos de Zalán (2011) em vermelho.
O primeiro passo da etapa 2 envolve calcular a topografia equivalente. O cálculo foi realizado com base em Kumar et al. (2011). O segundo passo envolve calcular a flexura $w(r)$ com base no modelo de flexura de placa fina.

A rigidez flexural é calculada utilizando uma janela média corrente de comprimento de $100 \mathrm{~km}$, com deslocamento de $20 \mathrm{~km}$. Valores padrões serão adotados para o Módulo de Young ( $E=100 \mathrm{GPa})$, para a razão de Poisson $\mathbf{v}=0.25$ e aceleração da gravidade $\left(9.81 \mathrm{~m} / \mathrm{s}^{2}\right)$. Os valores de densidade do manto $\left(3370 \mathrm{~kg} / \mathrm{m}^{3}\right)$ e densidade da crosta $\left(2880 \mathrm{~kg} / \mathrm{m}^{3}\right)$ são valores médios obtidos do modelo de densidade CRUST 2.0. A espessura elástica (Te) varia de 1 à $25 \mathrm{~km}$. Tais valores devem estar de acordo com a reologia local e são baseados em Tassara et al. (2007).

Os valores obtidos por análise flexural estão em concordância com a ICM gravimétrica, como pode ser visto na figura 2, que mostra a diferença entre as duas ICM. No geral, a diferença é pequena, variando de 2,5 a $3 \mathrm{~km}$. Os maiores valores estão próximos à área onde os resultados não são confiáveis (hachurada em cinza, na figura) e podem representar, ao menos em parte, influência desses dados.

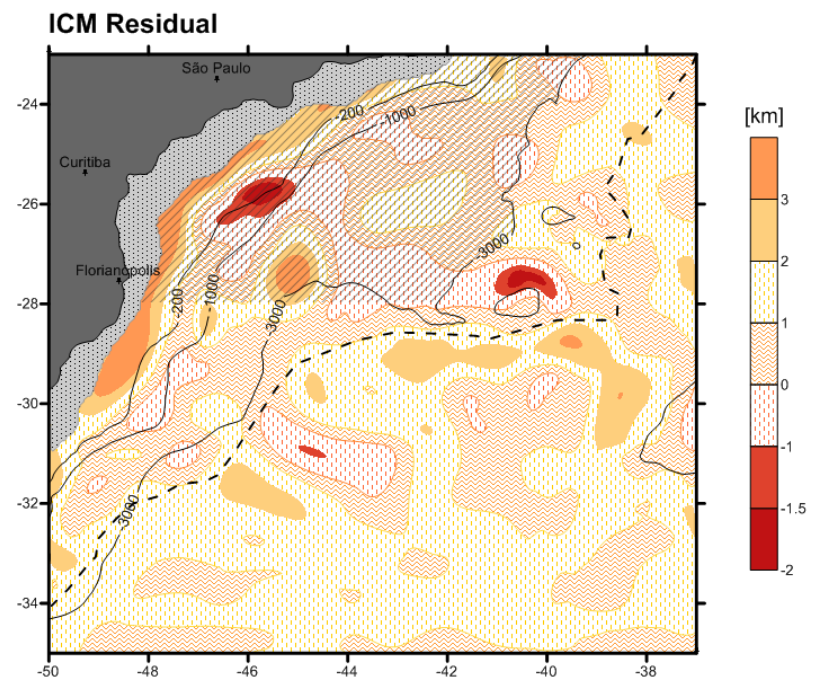

Figura 2: Diferença entre a ICM gravimétricae a ICM Flexural.

Na próxima etapa o efeito gravimétrico da ICM obtida na etapa 2 é calculado. Este efeito mais o efeito gravimétrico dos sedimentos são subtraídos na anomalia Bouguer, resultando no campo residual referente ao embasamento, o qual é invertido na última etapa deste trabalho.

Para a inversão do campo residual, foi utilizado um contraste de densidade constante, de $1570 \mathrm{~kg} / \mathrm{m}^{3}$, referente ao contraste de densidade entre a crosta superior $\left(2600 \mathrm{~kg} / \mathrm{m}^{3}\right)$ e a água $\left(1030 \mathrm{~kg} / \mathrm{m}^{3}\right)$. Todos os comprimentos de onda foram levados em consideração e a profundidade de referência, definida como o nível zero (Hwang 1999).

O termo embasamento utilizado neste trabalho refere-se à superfície física que se encontra abaixo da camada sedimentar. Os dados de espessura sedimentar utilizados neste trabalho representam a profundidade do embasamento acústico, definido como sendo o refletor 
observável mais profundo nos perfis de sísmica de reflexão e que pode não representar necessariamente a base dos sedimentos. O resultado final deste trabalho corresponde à base dos sedimentos e será chamado de embasamento gravimétrico (figura 3) Podemos observar depressões que chegam a $700 \mathrm{~m}$ de profundidade na área da Bacia de Santos.

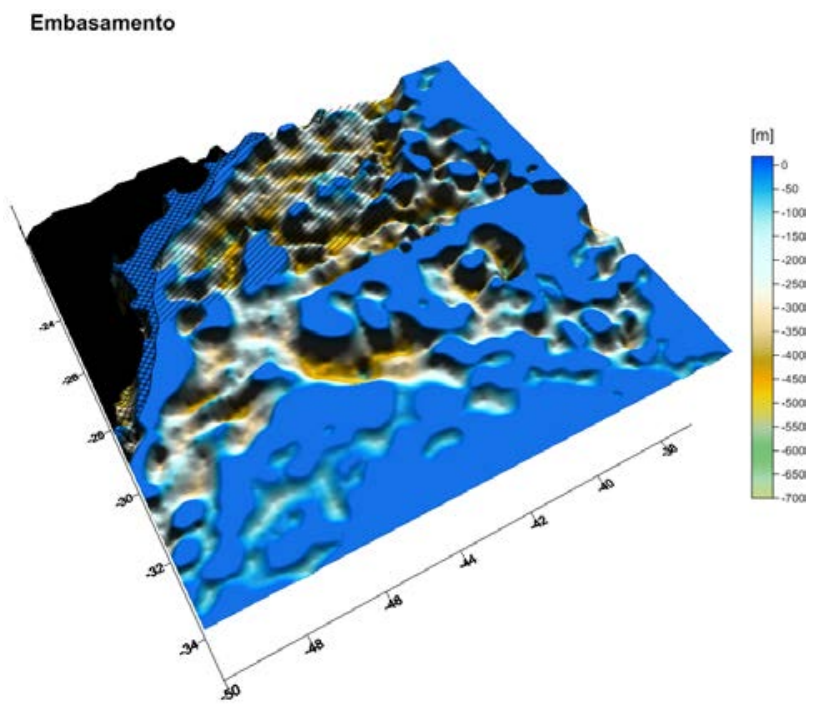

Figura 3: Embasamento gravimétrico, em metros.

A profundidade do embasamento é mostrada na figura 4. Os valores variam de 500 a 10500 metros.

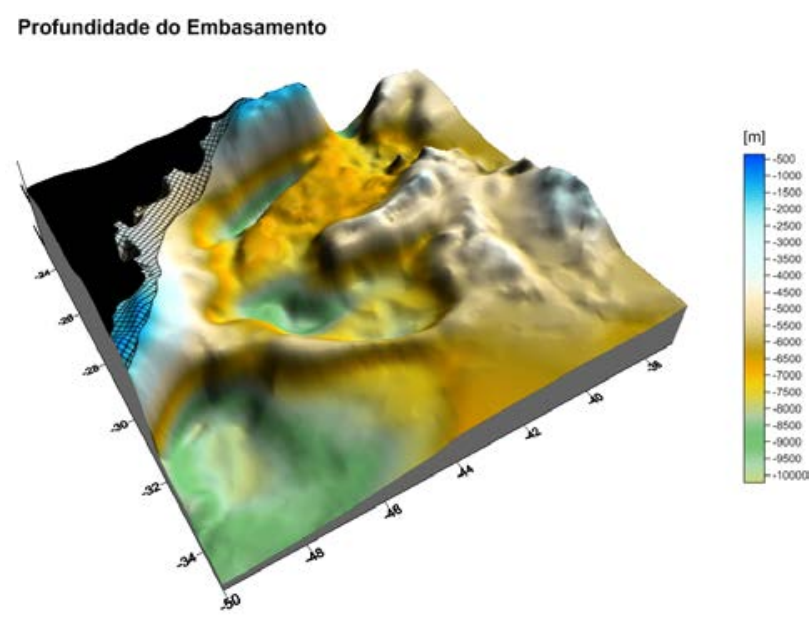

Figura 4: Profundidade do embasamento, em metros.

\section{Discussão e Conclusões}

Os resultados obtidos neste trabalho mostram que feições do embasamento encontram-se ocultas pela camada sedimentar na Região da Bacia de Santos. Para tanto, a topografia do embasamento foi determinada a partir dos dados disponíveis seguindo a metodologia proposta por Braitenberg et al. (2006).
A aplicação da primeira etapa desta metodologia permitiu a determinação da profundidade da Interface Crosta Manto (ICM) a partir da inversão do campo gravimétrico corrigido, utilizando como vínculos dados sísmicos obtidos por Leyden (1972) e Zalán (2011). Os resultados apresentaram um erro RMS de aproximadamente 1,9 km. Para checar a consistência dos resultados, a profundidade da ICM foi determinada através de um método independente, envolvendo análise flexural. Os valores, quando comparados aos anteriores, mostraram pequenas diferenças. A análise das maiores diferenças mostrou que estas podem estar associadas a intrusões salinas presentes entre as camadas de sedimentação da Bacia de Santos, descritas em literatura (Miesling et al., 2001; Izeli, 2008; Caldas \& Zalán, 2009; Souza et al., 2009).

Subtraindo do sinal gravimétrico observado o sinal gravimétrico da ICM e dos sedimentos, obteve-se o campo gravimétrico residual referente ao embasamento. O sinal gravimétrico dos sedimentos foi calculado considerando-se a sua compactação com base no modelo de Sclater e Christie (1980), com o sinal referente à ICM sendo calculado posteriormente pelo método de Parker (1972).

A inversão desse campo gravimétrico residual resultou na topografia do embasamento, que apresentou feições de destaque com até $700 \mathrm{~m}$, que parecem estar em concordância com feições discutidas em trabalhos pretéritos, como por exemplo a cadeia vulcânica Avedis (Meisling et al., 2001; Demercian, 1996).

A profundidade do embasamento estimada durante este trabalho mostrou profundidades que vão de 500 à $10500 m$. A região mais profunda do embasamento condiz com a falha de Cabo Frio (Macedo, 2004; Assine, 2008; Zalán 2009).

A metodologia utilizada durante este trabalho mostrou que é possível obter um modelo tridimensional do embasamento em áreas oceânicas a partir de dados gravimétricos obtidos por navio e por altimetria por satélite, dados batimétricos e informações sobre a espessura sedimentar e sobre a estrutura crustal da região. Este tipo de análise pode ser fornecer importantes subsídios para a exploração de hidrocarbonetos na região.

\section{Agradecimentos}

Ao CNPq, pela bolsa de mestrado relacionada a este projeto; a Carla Braitenberg pela cessão do software Lithoflex; a Andres Tassara pelo fornecimento dos dados de espessura elástica.

\section{Referências}

Braitenberg, C. e M. Zadro, (1999). Iterative 3D gravity inversion with integration of seismologic data, Bollettino Di Geofisica Teorica ed Applicata, v.40, n.3-4, p. 469-475.

Braitenberg, C., S. Wienecke, e Y. Wang, (2006). Basement structures from satellite-derived gravity field: 
South China Sea ridge, J. Geophys. Res., 111, B05407, doi:10.1029/2005JB003938.

Calmant, S., e N. Baudry, (1996). Modelling bathymetry by inverting satellite altimetry data: A review, Mar. Geophys. Res., 18, 123-134.

Hwang, C. (1999). A bathymetric model for the Souch China Sea from Satellite altimetry and depth data, Mar. Geod., 22,37-51.

Leyden, R. W.J. Ludwig e J. Ewing (1971). Structure of the continental margin of Punta del Este, Uruguay, and Rio de Janeiro, Brazil. AAPG Bulletin, 55: 2161-2173.

Molina, E.C., (2009). O uso de dados de missões geodésicas de altimetria por satélite e gravimetria marinha para a representação dos elementos do campo de gravidade terrestre. Tese de livre docência. Departamento de Geofísica do IAG-USP. Universidade de São Paulo. 100pp.

Parker, R. L. (1972). The rapid calculation of potential anomalies. Geophys. J. R. Astr. Soc., 31, 447-455.

Ramillien, G., e A. Cazenave (1996), Global bathymetry derived from altimeter data of the ERS-1 geodetic mission, J. Geodyn., 23, 129-149.

Russo, R. M., e R. C. Speed (1994), Spectral analysis of gravity anomalies and the architecture of tectonic wedging, NE Venezuela and Trinidad, Tectonics, 13(2), $613-622$.

Sclater, J. G. e P. A. F. Christie (1980), Continental stretching: An explanation of the post mid-Cretaceous subsidence of the central North Sea basin, J. Geophys. Res., 85, $3711-3739$.

Smith, W. H. F., e D. T. Sandwell (1997), Global sea floor topography from satellite altimetry and ship depth soundings, Science, 277, 1956-1962.

Woodward, D. e R. Wood, (2000), Bathymetry sediment thickness and crustal structure from satellite gravity data, $\begin{array}{llll}\text { Exploration Geophysics } & 31(2) \quad 89 & - & 93\end{array}$ doi:10.1071/EG00089

Zalán, P.V., M.C.G. Severino, C.A. Rigoti, L.P. Magnaviva, J.A.B. Oliveira e A.R. Vianna, (2011), An Entirely New 3D-View of the Crustal and Mantle Structure of a South Atlantic Passive Margin - Santos, Campos and Espírito Santo Basins, Brazil, Search and Discovery Article \#30177 